\title{
Exact Solutions with Lie Symmetry Analysis for Nano-Ionic Currents along Microtubules
}

\author{
Zühal Küçükarslan Yüzbaş1 ${ }^{1, *}$, Ebru Cavlak Aslan ${ }^{1}$, and Mustafa Inc ${ }^{1}$ \\ ${ }^{1}$ Firat University, Faculty of Science, Department of Mathematics, 23119 Elazig / Turkey
}

\begin{abstract}
In this work, the Lie symmetry analysis is tested by its applications to nano-solitons of ionic waves propagation along microtubules in living cells and nano-ionic currents of MTs.
\end{abstract}

\section{Introduction}

The main aim of this work is to study the executive equation defining nano-ionic currents along microtubules with the Lie symmetry analysis. This method is one of the most important and efficient techniques to find the exact solutions [1]-[7].

The nano ionic currents are elaborated in [8] take the form

$$
\frac{L^{2}}{2} q_{x x x}+\frac{Z^{3 / 2}}{L}\left(\chi G_{0}-2 \delta C_{0}\right) q q_{t}+2 q_{x}+\frac{Z C_{0}}{L} q_{t}+\frac{1}{L}\left(R Z^{-1}-G_{0} Z\right) q=0,
$$

where $R=0.34 \times 10^{9} \Omega$ the resistance of the elementary rings (ER), $L=8 \times 10^{-9} \mathrm{~m}, C_{0}=$ $1.8 \times 10^{-15} \mathrm{~F}$ is the total maximal capacitance of the ER. $G_{0}=1.1 \times 10^{-13} \mathrm{Si}$ is the conductance of pertaining nano-pores (NPS) and $Z=5.5 \times 10^{10} \Omega$ is the characteristic impedance of the system. The parameter $\delta$ and $\chi$ describe the nonlinearity of ER capacitor and conductance of NPS in ER, respectively.

\section{Lie Symmetry Analysis}

We will now consider the one parameter group of point transformation of the form

$$
\begin{aligned}
& x \rightarrow x+\varepsilon \xi(x, t, q), \\
& t \rightarrow t+\varepsilon \tau(x, t, q), \\
& q \rightarrow q+\varepsilon \phi(x, t, q),
\end{aligned}
$$

where $\varepsilon<1$ is a group parameter [9]. The vector field associated with the above group of transformations can be written as

$$
V=\xi(x, t, q) \frac{\partial}{\partial x}+\tau(x, t, q) \frac{\partial}{\partial t}+\phi(x, t, q) \frac{\partial}{\partial q} .
$$

\footnotetext{
*Corresponding Author: zuhal2387@yahoo.com.tr
} 
In order to study symmetries, from Eq. (1), it can be shown by a well established procedure as following

$$
\begin{aligned}
\xi & =C_{2}, \\
\tau & =C_{1}, \\
\phi & =0,
\end{aligned}
$$

where $C_{1}$ and $C_{2}$ are constants. The Lie symmetry algebra of Eq. (1) is spanned by the following two generators.

$$
\begin{aligned}
& V_{1}=\frac{\partial}{\partial x}, \\
& V_{2}=\frac{\partial}{\partial t} .
\end{aligned}
$$

Now we consider the above mentioned vector fields for Eq. (1).

Case 1: For the generator $V_{1}=\frac{\partial}{\partial x}$, the similarity variables are $\eta=t, q=f(\eta)$. Then from Eq. (1), we get the following reduction equation

$$
\frac{Z^{3 / 2}}{L}\left(\chi G_{0}-2 \delta C_{0}\right) f f^{\prime}+\frac{Z C_{0}}{L} f^{\prime}+\frac{1}{L}\left(R Z^{-1}-G_{0} Z\right) f=0
$$

Solution of this equation

$$
f(\eta)=\frac{C_{0}}{Z^{1 / 2}\left(\chi G_{0}-2 \delta C_{0}\right)} \text { Product } \log \left[\frac{Z^{1 / 2}\left(\chi G_{0}-2 \delta C_{0}\right)}{C_{0}} e^{\alpha \eta+C}\right],
$$

where $\alpha=\left(R Z^{-1}-G_{0} Z\right) / Z C_{0}$.

Case 2: For the generator $V_{2}=\frac{\partial}{\partial t}$, the similarity variables are $\eta=x, q=f(\eta)$. Then from Eq. (1), we get the following reduction linear equation

$$
\frac{L^{2}}{3} f^{\prime \prime \prime}+2 f^{\prime}+\frac{1}{L}\left(R Z^{-1}-G_{0} Z\right) f=0
$$

Case 3: For the generator $V_{3}=V_{1}+V_{2}=\partial / \partial x+\partial / \partial t$, we have $q=f(\eta)$ that $\eta=x-t$. If we substitute $q=f(\eta)$ into the governing equation, we obtain following NODE;

$$
\frac{L^{2}}{3} f^{\prime \prime \prime}-\frac{Z^{3 / 2}}{L}\left(\chi G_{0}-2 \delta C_{0}\right) f f^{\prime}+\left(\frac{2 L-Z C_{0}}{L}\right) f^{\prime}+\frac{1}{L}\left(R Z^{-1}-G_{0} Z\right) f=0 .
$$

Case 4: For the infinitesimal generator $V_{4}=\frac{1}{\lambda} \frac{\partial}{\partial x}+\frac{1}{\rho} \frac{\partial}{\partial t}$, we have $q=f(\eta), \eta=\lambda x-\rho t$ where $\lambda$ and $\rho$ are constants. If we substitute $q=f(\eta)$ into Eq. (1), so that

$$
\frac{L^{2}}{2} \lambda^{3} f^{\prime \prime \prime}-\frac{Z^{3 / 2}}{L}\left(\chi G_{0}-2 \delta C_{0}\right) \rho f f^{\prime}+\left(2 \lambda-\frac{Z C_{0}}{L} \rho\right) f^{\prime}+\frac{1}{L}\left(R Z^{-1}-G_{0} Z\right) f=0 .
$$

In this, we find the solution of Eq. (7) using the "Generalized Projective Riccati Equations Method" (GPREM) [10]. So, balancing $f^{\prime \prime \prime}$ and $f f^{\prime}$ gives $N=2$. Therefore

$$
f(\eta)=A_{0}+\sum_{i=1}^{2} \sigma^{i-1}\left[A_{i} \sigma(\eta)+B_{i} v(\eta)\right]
$$

where $A_{j}$ and $B_{j}, j=0,1,2$ are constants. 
In Eq. (8), $\sigma(\eta)$ and $v(\eta)$ satisfy the ODE

$$
\begin{gathered}
\sigma^{\prime}=\epsilon \sigma(\eta) v(\eta), \\
v^{\prime}=R+\epsilon v^{2}(\eta)-\mu \sigma(\eta), \epsilon= \pm 1,
\end{gathered}
$$

where

$$
v^{2}=\epsilon\left(R-2 \mu \sigma(\eta)+\frac{\mu^{2}+r}{R} \sigma^{2}(\eta),\right.
$$

$r, R$ and $\mu$ are non zero constants.

So, if Eq. (8) are written in Eq. (7) for $\epsilon=-1$ and $r=1$, we get solving the system of the algebraic equation.

Consequently, the solution of $\mathrm{f}(\mathrm{z})$ is obtained as following

$$
f(z)=-\frac{3 \lambda^{2}}{2 \alpha_{2} \rho} \frac{\csc h\left[z \sqrt{\frac{-i}{\lambda \alpha_{1}}}\right]}{\left(1+i \csc h\left[z \sqrt{\frac{-i}{\lambda \alpha_{1}}}\right]\right)}+\frac{2 \lambda-i \lambda^{2}-\rho \alpha_{3}}{\alpha_{2} \rho}
$$

where $\alpha_{1}=\frac{L^{2}}{2} \lambda^{3}, \alpha_{2}=\frac{Z^{3 / 2}}{L}\left(\chi G_{0}-2 \delta C_{0}\right) \rho, \alpha_{3}=\left(2 \lambda-\frac{Z C_{0}}{L} \rho\right)$ and finally we get $\alpha_{4}=$ $\frac{1}{L}\left(R Z^{-1}-G_{0} Z\right)$.

\section{Conclusion}

In this work, the Lie symmetry analysis has been successfully applied to find the solution of the nonlinear partial differential equation which plays an important role in biology. Some of the equations obtained with the Lie symmetry are solved and we found new exact solutions for these equations. Also, while finding these new exact solutions, Generalized Projective Riccati method was applied to the reduced equation by Lie analysis.

\section{References}

[1] M. Younis and S. Ali, Solitary wave and shock wave solitons to the transmission line model for nano-ionic currents along microtubules, Appl. Math. Comput., 246, 460-463, 2014.

[2] M. Younis and S. T. R. Rizvi, Analytical and soliton solutions: Nonlinear model of nanobioelectronics transmission lines, Appl. Math. Comput., 265, 994-1002, 2015.

[3] D. Baleanu, M. Inc, A. Yusuf and A. I. Aliyu, Lie symmetry analysis, exact solutions and conservation laws for the time fractional modified Zakharov Kuznetsov equation, Nonlinear Anal. Model. Control, 22, 6, 861-876, 2017.

[4] M. Inc, A. I. Aliyu and A. Yusuf, Solitons and conservation laws to the resonance nonlinear Shrödinger equation with both spatio-temporal and inter-modal dispersions, Optik, 142, 509-522, 2017.

[5] D. Baleanu, M. Inc, A. Yusuf and A. I. Aliyu, Lie symmetry analysis, exact solutions and conservation laws for the time fractional Caudrey- Dadd- Gibbon- Sawada- Kotera equation, Commun. Nonlinear Sci. Numer. Simul., 59, 222-234, 2018.

[6] D. Baleanu, M. Inc, A. Yusuf and A. I. Aliyu, Time Fractional Third-Order Evolution Equation: Symmetry Analysis, Explicit Solutions, and Conservation Laws, J. Comput. Nonlinear Dynam, 13(2), 021011, 2017. 
[7] M. Inc, A. I. Aliyu, A. Yusuf and D. Baleanu, Optical solitons and modulation instability analysis with $(3+1)$-dimensional nonlinear Shrödinger equation, Superlattices and Microstructures, 112, 296-302, 2017.

[8] D. L. Sekulic, M. V. Sataric and M. B. Zivanov, Symbolic computation of some new nonlinear partial differential equations of nanobiosciences using modified extended tanh-function method, Appl. Math. Comput., 218, 3499-3506, 2011.

[9] T. Ozis and I. Aslan, Simirity Solutions to Burgers' Equation in terms of Special Functions of Mathematical Physics, Acta Phy. Pol. B, 48, 1349-1369, 2017.

[10] M. Inc, A. I. Aliyu and A. Yusuf, Solitons and conservation laws to the resonance nonlinear Shrdingers equation with both spatio-temporal and inter-modal dispersions, Optik, 142, 59-522, 2017. 